\title{
Rendimiento escolar en matemáticas de estudiantes de primaria en el municipio de Tibú Norte de Santander
}

\author{
School performance in mathematics of primary school students in the \\ municipality of Tibú Norte de Santander \\ José Trinidad Pérez-Cabrales ${ }^{\mathrm{a}}$

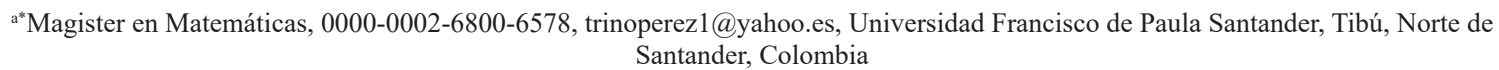

Forma de citar: Pérez-Cabrales, J.T. Rendimiento escolar en matemáticas de estudiantes de primaria en el municipio de Tibú Norte de Santander. Eco Matemático, 10 (2), 54-64

Recibido: 2 marzo 2019

Aceptado: 4 mayo 2019

\section{Palabras clave}

Rendimiento escolar, factor socio económicos, factor cultural, aprendizaje de las matemáticas

*Autor para correspondencia trinoperez1@yahoo.es

http://dx.doi.org/10.1016/j.eq/

2590-9215 C 2017 Universidad Francisco de Paula Santander. Este es un artículo bajo la licencia CCBYNCND

Resumen:Este proyecto de investigación tiene como propósito indagar sobre los factores sociales económicos, políticos y culturales asociados en el aprendizaje de las matemáticas en estudiantes de básica primaria del municipio de Tibú como zona de conflicto armado.

La investigación realizada se hizo con un enfoque cuantitativo, de tipo descriptivo, En la cual participaron 47 estudiantes 21 padres de familia y 10 docentes. A los participantes se les aplicó una entrevista enfatizando los factores sociales, económicos y culturales que inciden el rendimiento escolar de los estudiantes.

Dentro de los resultados se evidencia que: los estudiantes son utilizados para realizar trabajos en el campo, además no reciben el apoyo emocional y económico de sus padres en la orientación de sus tareas y la compra de los implementos educativos necesario para el desarrollo de su actividad académica. En cuanto a los docentes del área, no están motivados y por lo tanto no motivan a sus estudiantes y el desarrollo de sus clases son monótonas y aburridas. Los padres de familia en su mayoría son de muy de bajo recursos económicos y su grado de escolaridad es baja. De lo anterior podemos concluir que: existen factores socio-económicos que influyen en el rendimiento escolar de los estudiantes, en el cual están involucrados los docentes, que se siente poco apreciaos por la comunidad educativa, por otra parte, los estudiantes no cuentan con el apoyo y la motivación de los padres y docentes. 


\section{Keywords \\ School performance, Socioeconomic factor, Cultural factor, Math learning}

\begin{abstract}
The purpose of this research project is to investigate the social, economic, political and cultural factors associated with the learning of mathematics in elementary school students in the municipality of Tibú as an area of armed conflict.

The research was carried out with a quantitative, descriptive approach, in which 47 student 21 parent and 10 teachers participated. Participants were given an interview emphasizing the social, economic and cultural factors that affect students' school performance.

Among the results it is evident that: students are used to carry out work in the field, they also do not receive the emotional and economic support of their parents in the orientation of their tasks and the purchase of the educational implements necessary for the development of their activity academic As for the teachers in the area, they are not motivated and therefore do not motivate their students and the development of their classes are monotonous and boring. The majority of parents are of very low economic resources and their level of schooling is low. From the above we can conclude that: there are socioeconomic factors that influence the students' school performance, in which teachers are involved, which is not appreciated by the educational community, on the other hand, students do not have the support and the motivation of parents and teachers.
\end{abstract}

\section{Introducción}

En la enseñanza de las matemáticas se debe estimar y valorar todos aquellos aspectos que de alguna manera intervienen en este proceso, con el objetivo de establecer criterios que conlleven a elaborar propuestas didácticas y que contribuyan al aprendizaje de esta área, enfocados en el estudiante como centro de la actividad pedagógicas, pero ligado estrechamente con la labor docente, pues algunos especialistas argumentan que es tan importante lo que se enseña, como y quien lo realiza, para ello se debe conocer los factores que intervienen en el aprendizaje de las matemáticas.

Los resultados de las evaluaciones externas e internas en el área de matemáticas de los estudiantes de básica primaria del centro educativo Bertrania han sido bajos en los últimos años, además la apatía y el poco interés de los estudiantes es otro de los factores preocupante y es por ello se pretende identificar los factores asociados al rendimiento escolar
El Centro Educativo Rural Bertrania ha venido mejoran levemente en los resultados del índice sintético de calidad en los últimos años, pero se encuentra en un nivel más bajo del promedio nacional local, específicamente en el área de matemáticas en básica primaria,

Es notorio ver en las pruebas internas los educandos reflejan apatía y desmotivación, para ello es lo mismo sacar buenos resultados o perderla, además, una gran mayoría no realizan las actividades extraescolares y además la participación en clase es casi inexistente, sus actitudes con respecto a las matemáticas son de desmotivación, desinterés. Por otro lado, los padres y acudiente tampoco demuestras preocupación aparente, pareciera que solo les interesara que sus hijos estén el colegio para recibir el subsidio que brinda el estado. Según argumentan los estudiantes, las clases de matemáticas, son aburridas y rutinarias, hasta el punto de no querer hacer parte de ella. Por tal motivo esta problemática sumada 
al bajo rendimiento escolar y a la preocupación de los docentes, directivos docentes y algunos padres o acudientes, es el principal motivo de la investigación, esto nos ha llevado a indagar sobre los factores que inciden en el bajo rendimiento escolar. Esto suscita una serie de cuestionamientos en la cual se han intentado buscar dar respuesta a estos cuestionamientos como son: dificultad de aprendizaje, factores genéticos y métodos de enseñanza, los hábitos de estudio, las motivaciones, las actitudes de los estudiantes, etc. (Núñez et al., 2005; Carbonero y Navarro, 2006). Teniendo en cuenta lo anterior, mi aspiración fundamental en esta investigación es conocer la incidencia que tienen algunos de esos factores (actitudes de los estudiantes hábitos de estudio, situación socio económica y el acompañamiento, motivación de sus padres) sobre su rendimiento en Matemáticas. Existe un sin número de investigaciones dedicadas a establecer los factores que inciden en el rendimiento académico de los estudiantes se ha basado en las variables sociales, económicas y culturales, como también la escolaridad de los padres, número de hijos etc. (Casanova, Cruz, de la Torre \& de la Villa, 2005; Eamon, 2005; Jones \& White, 2000). Aunque es de vital importancia conocer los factores que inciden en el rendimiento de los estudiantes, algunos de ellos resultan difíciles de modificar como son el bajo nivel económico y el grado de escolaridad de los padres y acudientes, pero se cuenta la base estadística para diseñar estrategias a ser tenidas en cuentas en las futuras generaciones.

El rendimiento académico es la capacidad que tiene un individuo para interiorizar los contenidos académicos en relación a estímulos, objetivos y propósitos educativos previamente establecidos. Cuando un estudiante presenta un rendimiento académico bajo, nos da a conocer que el estudiante no ha interiorizado los conocimientos académicos, por ende, no posee la habilidad y la capacidad necesarias para la solución de problemas presentados en su entorno y en su vivencia cotidiana, esto lleva al estudiante al fracaso del educando y a la deserción escolar, realidad que afecta a toda la comunidad educativa. La incidencia de factores asociados al rendimiento académico que no son corregidos y permanecen sin ser corregidos causa dificultades en la memoria, atención y concentración, y bajo rendimiento escolar.

Entrelasvariables presentadasenlainvestigación en los estudiantes podemos las habilidades de estudio, la motivación de los estudiantes, y el acompañamiento por parte de los padres, según (Lammers, Onweugbuzie \& Slate, 2001; Proctor, Hurst, Prevatt, Petscher \& Adams, 2006) El éxito de los estudiantes se basa en las habilidades que presente el para aprender y su disposición para interiorizar los nuevos conocimientos, los hábitos de estudio y la situación laboral de los niños, ya que en su mayoría son utilizados para realizar trabajos en el campo. Aunque no se ha demostrado específicamente si la motivación de los estudiantes promueve que dediquen más tiempo y utilicen mejores estrategias de aprendizaje y adquieran habilidades de aprendizaje, pero es notorio ver el efecto positivo en el rendimiento escolar en los niños. Caso-Niebla, Joaquín; (Hernández-Guzmán, Laura Variables que inciden en el rendimiento académico de adolescentes mexicanos, 2007 pag 489.) Esto demuestra que un estudiante motivado permanece más activo y dispuesto a recibir y asimilar el nuevo conocimiento, la motivación hace que el estudiante este activo, concentrado y su capacidad cognitiva esté funcionando con más eficacia, por tanto, su capacidad de aprendizaje es más eficaz. De acuerdo con otras posturas, como la de Shavelson, Hubner y Stanton (1976), se integraría también en dos dimensiones, académica y una no académica, pero reconocen la existencia de componentes emocionales, sociales y físicos (personales) de la autoestima no académica. Tomado de Joaquín Caso Niebla, Laura Hernández-Guzmán. (2010)

De entre las variables personales comúnmente asociadas con el rendimiento académico se encuentran las habilidades de estudio. Esta relación 
se ha documentado ampliamente, y sugiere la contribución de éstas a la explicación del éxito fracaso escolar y a la caracterización de estudiantes con alto y bajo rendimiento (Lammers, Onweugbuzie \& Slate, 2001; Proctor, Hurst, Prevatt, Petscher \& Adams, 2006). La organización y concentración en el estudio, la capacidad para relacionar nuevos conocimientos con los existentes, la comprensión lectora y la capacidad para autorregular el aprendizaje, son habilidades que correlacionan con el rendimiento académico de los estudiantes (Lammers et al., 2001; Ruban, 2000; Valle, González, Núñez y González-Pienda, (1998) Tomado de Caso-Niebla, Joaquín; Hernández-Guzmán, Laura. (2008). Para (Lupart, Cannon \& Telfer 2004). En el contexto del desarrollo adolescente, el establecimiento de metas se ha asociado con el rendimiento académico, la orientación vocacional y la educación para el trabajo. Lammers et al., 2001; Valle, Gonzáles Núñez Gonzáles Pineda. Afirma que la organización y la concentración en el estudio, la capacidad de relacionar los nuevos conocimientos con los que ya existen, la capacidad de leer y la capacidad para regular el aprendizaje son habilidades que se correlacionan con el rendimiento académico de los estudiantes.

Otras de las variables asociadas al rendimiento académico es la variable distal como el nivel socioeconómico, la escolaridad de los padres, el tipo de institución educativa, el barrio de residencia, etc. (Casanova, Cruz, de la Torre \& de la Villa, 2005; Eamon, 2005; Jones \& White, 2000). Aunque se determine la influencia de esta variable en el rendimiento.

Importante tenerlas en cuenta y concientizar a las personas involucradas para que se personalicen en esta situación. Según. (Pedro F. Casanova, M. Cruz García-Linares, Manuel J. de la Torre and M. de la Villa Carpio) propuso una metodología para clasificar a los padres en cuatro categorías según sus niveles: El estilo educativo de los padres, el comportamiento específico de los padres y las variables de aceptación y control. Para Laura Desimone, (1999). La participación de los padres en el aprendizaje de los niños en la escuela y en el hogar se considera un componente clave de la reforma escolar, pero se necesita más información sobre cómo varían los efectos de esta participación para los estudiantes de diferentes orígenes étnicos y económicos. La participación de los padres de familia con su hijo, participación que está ligada al interés que demuestran los padres por el rendimiento académico de sus hijos y la motivación que ejerzan sobre ellos.

Se ha demostrado la relación que existe entre dimensión parental y el rendimiento académico a través del nivel sociocultural y estructural de la familia, Stevenson y Baker (1987) confirmo que el rendimiento académico de los niños está relacionado con el nivel de participación que tenga con sus hijos. (Lammers, Onwenugbuzie \& slate, 2001; Protor, Huest, Prevatt, Petscher \&Adams, 2006) La organización y concentración en el estudio, la capacidad para relacionar nuevos conocimientos. Tomado de Caso Niebla J, Hernández-Guzmán L (2010) pág. 448.

Por otro lado, Campo Arias et al. (2005) mencionan que otros estudios no han encontrado diferencias significativas entre el rendimiento escolar entre grupos con depresión y sin depresión, además de plantear la importancia de discriminar otros factores que pudieran estar asociados al bajo rendimiento escolar. En este sentido, se ha identificado que el nivel socioeconómico es un factor que incide en el aprovechamiento escolar. Tomado de, Galicia Moyeda I, Sánchez Velasco y Francisco Javier Robles O Jeda. 2009.

Además, para García-Quintero, C., \& Villamizar Suárez, G. (2017). “El método fenomenológico permite establecer las concepciones que sobre la experiencia evaluativa vivida a lo largo de años de formación de los sujetos, la convierten en parte 
sustancial de la conciencia tanto de docentes como de estudiantes"

\section{Método}

Se plantean algunas definiciones de conceptos relevantes en la investigación según el puno de vista de diferentes teóricos. Cada uno de ellos será explorado desde diferentes autores para construir una definición propia. Los términos analizados son las bases conceptuales que enmarcan la investigación aspectos relevantes para el proyecto. Todos ellos relacionados con los temas en los que se concentra la investigación.

Este proyecto investigativo se basa en la teoría constructivista, de acuerdo a los enfoques conceptuales de Jean Piaget, Vygotsky y Ausbel $\mathrm{y}$ algunas consideraciones teóricas y propuestas tenidas en cuenta por Bruner, ya que el centro educativo donde se llevó a cabo la investigación trabaja con metodología activa, con un modelo constructivista, que son modelo que mantiene que una persona, tanto en los aspectos cognitivos, sociales y afectivos del comportamiento, no es un mero producto del ambiente ni un simple resultado de sus disposiciones internas, sino un conjunto de estrategias didácticas donde el estudiante genera su propio conocimiento a través de la práctica y la experimentación como resultado de la interacción de estos dos factores. En consecuencia, según la posición constructivista, el conocimiento no es una copia de la realidad, sino una construcción del ser humano basados en los conocimientos previos en relación al ambiente que lo rodea.

La presente investigación se realiza mediante el método descriptivo explicativo con enfoque cuantitativo basado en el estudio y análisis de la realidad a través de diferentes procedimientos de medición, utilizando como instrumento de recolección de la información la encuesta y la observación directa. Con esta investigación se pretende conocer los factores inciden en el rendimiento académico de los niños, padres de familia y docentes del Centro Educativo Rural Bertrania Municipio de Tibú, Esta investigación se realiza en la sede principal, por tener mayor número de estudiantes y la que presenta la mayor población estudiantil con dificultades de aprendizaje en el área de las matemáticas; Aunque en su mayoría las escuelas rurales del C.E.R. Y en general del municipio, presentan esta tendencia.

El Centro Educativo Rural Bertrania sede principal Posee una población estudiantil de 327 niños y adolescentes matriculados en el SIMAT. De los cuales se tomó como muestra los 47 estudiantes de Cuarto y quinto grado de educación básica, que comparten salón de clase, debido al aumento de la población estudiantil en los últimos años con la llegada de familias desplazadas del vecino país por la violencia que azota la región, de esta muestra el $51.2 \%$ corresponde al sexo Femenino, y el $48.8 \%$ al sexo masculino, con edades comprendidas entre 8 a 14 años, se consideró como unidad de muestra a los grupos de estudiantes de los grados mencionados anteriormente.

\section{Instrumento}

El instrumento utilizado para la recolección de datos corresponde a una encuesta aplicada a 45 estudiantes del sector rural que cursan cuarto y quinto grado de básica primaria en el centro educativo Rural Bertrania municipio de Tibú, también se le aplicó una encuetas a 10 docentes que laboran en este centro educativo en el área de matemáticas y a 22 padres y acudientes de los niños que se les aplicó la encuetas.

La encuesta estaba estructurada en una serie de preguntas diseñadas a recolectar información relacionada con la caracterización de los encuetados, teniendo en cuenta aspectos relevantes de su vida que pueden influir y conformar un factor que incide 
en la enseñanza y el aprendizaje de esta área. Cada pregunta cuenta con cinco opciones de respuestas según la información y las vivencias de cada individuo.

Las preguntas estaban estructuradas de acuerdo a las siguientes variables: Factores internos, de ellos se investigó , la pedagogía utilizada por los docentes en su quehacer educativo, la enseñanza, métodos y estrategias utilizada por los docentes, los elementos que intervienen en el aprendizaje de los estudiante, factores externos como: la convivencia escolar dentro y fuera del aula y la familias, su aporte en el proceso de la educación de sus hijos, también se investigó los factores socioeconómicos que juegan un papel determinante en la educación.

\section{Análisis estadístico}

Se realizaron los siguientes tipos de análisis:

a) Tabulación de datos

b) obtención de estadísticos básicos,

c) Análisis comparativo y discusión analítica de cada ítem encuestado.

\section{Resultado}

Una vez aplicada la encuesta, se procedió a la tabulación y análisis de los resultados.

La encuesta aplicada a los docentes constaba de una caracterización, teniendo en cuenta los aspectos más relevantes de cada docente a nivel personal, que tu ver incidencia en la enseñanza, además se plantearon 40 preguntas distribuidas en cuatro grandes categorías. (Prácticas pedagógicas, ambientes de aprendizaje, proceso de comunicación y actitudes sobre las matemáticas). Cada categoría contenía una serie de preguntas basadas en los aspectos más influyentes, en los cuales el docente a través de la reflexión de su praxis, nos proporcionaba la información necesaria para la investigación.
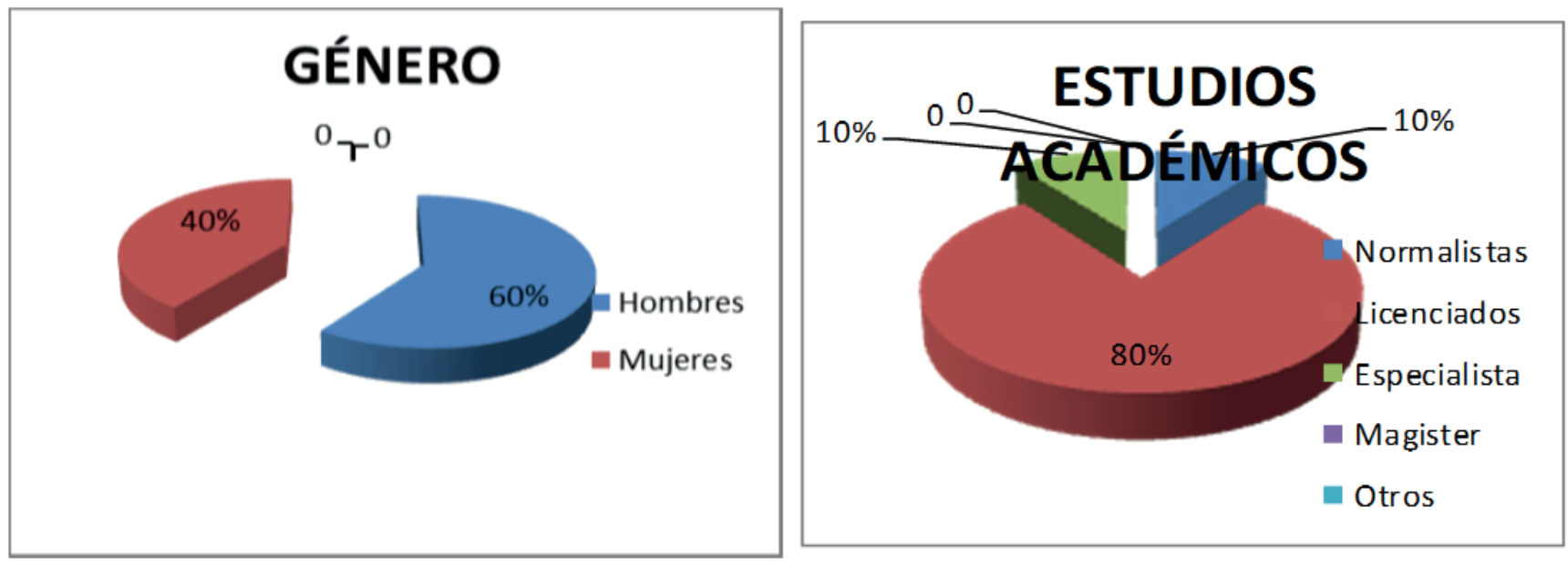

Gráfica 1. Caracterización de los docentes del área de matemáticas 


\section{Ítem}

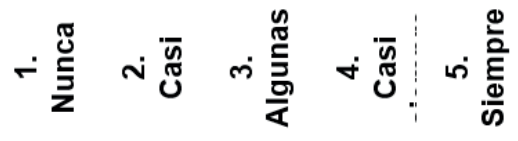

\begin{tabular}{|c|c|c|c|c|c|c|}
\hline 12 & $\begin{array}{l}\text { Propone actividades de reflexión a los estudiantes sobre los } \\
\text { nuevos conocimientos asimilados }\end{array}$ & 0 & 0 & $20 \%$ & $40 \%$ & $40 \%$ \\
\hline 34 & Realiza la misma rutina de clase & 0 & $20 \%$ & $60 \%$ & $20 \%$ & 0 \\
\hline 35 & $\begin{array}{l}\text { Realiza planificación curricular y colaborativa con otros } \\
\text { docentes de área }\end{array}$ & 0 & $30 \%$ & 0 & $50 \%$ & $20 \%$ \\
\hline 40 & $\begin{array}{l}\text { A partir de los resultados de evaluación de desempeño docente } \\
\text { se toman decisiones pedagógicas para la mejora de las } \\
\text { prácticas. }\end{array}$ & & & $10 \%$ & $60 \%$ & $30 \%$ \\
\hline
\end{tabular}

Graficas 2. Aspectos más importantes de la encuesta a los docentes del área de matemáticas

A los estudiantes de cuarto y quinto de básica primaria del centro educativo se les aplicó una encuesta similar, que constaba de una caracterización (datos personales relevantes para la investigación) y 47 pregunta correspondiente a cinco categorías. (Enseñanza, aprendizaje, convivencia escolar, aspectos familiares y aspectos económicos). En esta encuesta los estudiantes dejaron ver aspectos que son transcendentes en la investigación.

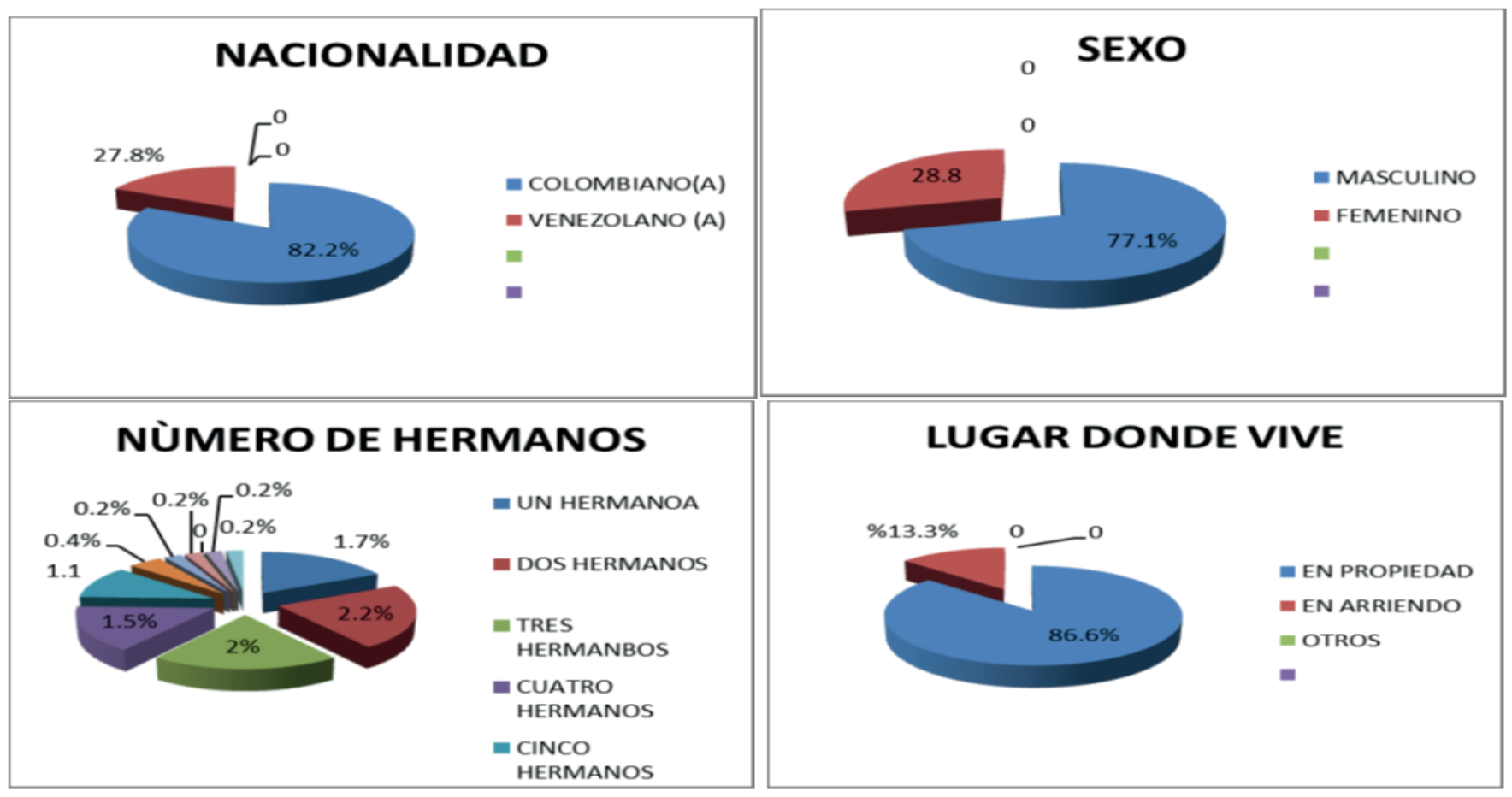

Gráfica 3. de Caracterización de los estudiantes cuarto y quinto grado 


\begin{tabular}{|c|c|c|c|c|c|c|}
\hline & Ítem &. & $\overline{0}$ & 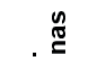 & $\bar{n}$ & 을 \\
\hline $\begin{array}{l}5 \\
8\end{array}$ & $\begin{array}{l}\text { El docente de matemáticas realiza actividades en clase que } \\
\text { despiertan tu interés por aprender. }\end{array}$ & $20 \%$ & $8.8 \%$ & $\begin{array}{l}28.8 \\
\%\end{array}$ & $\begin{array}{l}8.8 \\
\%\end{array}$ & $\begin{array}{l}33.3 \\
\%\end{array}$ \\
\hline 62 & El ambiente del aula de clase es agradable y armónico & $15 \%$ & $20 \%$ & $\begin{array}{l}6.6 \\
\%\end{array}$ & $\begin{array}{c}17.7 \\
\%\end{array}$ & $40 \%$ \\
\hline 63 & $\begin{array}{l}\text { El docente de matemáticas tiene dominio del tema, es } \\
\text { receptivo y agradable }\end{array}$ & 24.4 & 13.3 & 22.2 & 26.6 & 17.7 \\
\hline 69 & $\begin{array}{l}\text { Las condiciones físicas del aula de clase son apropiadas } \\
\text { (Iluminación ventilación espacio y ruido) }\end{array}$ & $22.2 \%$ & $22.2 \%$ & $\begin{array}{l}17.7 \\
\%\end{array}$ & $\begin{array}{l}8.8 \\
\%\end{array}$ & $\begin{array}{l}33.3 \\
\%\end{array}$ \\
\hline 72 & Te sientes feliz cuando inicia la clase de matemáticas. & $40 \%$ & $6.6 \%$ & $\begin{array}{l}11.1 \\
\%\end{array}$ & $\begin{array}{l}17.7 \\
\%\end{array}$ & $\begin{array}{l}24.4 \\
\%\end{array}$ \\
\hline 74 & Motivación del grupo & $31 \%$ & $20 \%$ & $\begin{array}{l}28.8 \\
\%\end{array}$ & $\begin{array}{l}11.1 \\
\%\end{array}$ & $\begin{array}{l}8.8 \\
\%\end{array}$ \\
\hline
\end{tabular}

Gráfica 4. Datos más relevantes de la encuesta aplicada a los estudiantes

También se les aplicó una encuesta a 22 padres de familia, que constaba de unas preguntas de caracterización de los padres y acudientes y 20 preguntas relacionadas con la motivación, participación compromiso, y dedicación en la enseñanza y aprendizaje de sus hijos, de los cuales los más relevantes tiene que ver con

Poco interés por conocer el rendimiento y comportamiento de su hijo en el colegio, poca vinculación y colaboración en asesorar a los niños en las tareas y revisión de sus compromisos escolares.

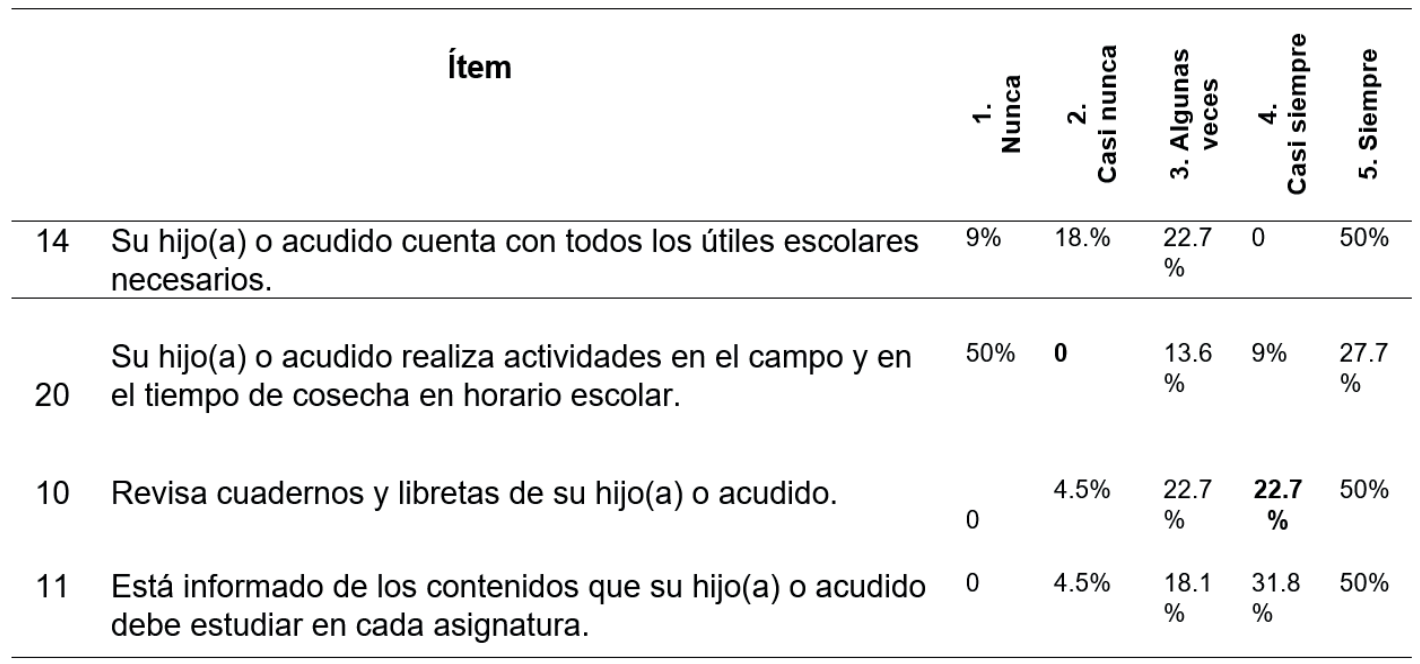

Graficas 5. Aspectos más importantes de la caracterización de los padres de familias y acudiente 
Basado en esta investigación corroboro los resultados de diversos estudios realizados en este campo. En el trabajo de investigación identifican las principales variables y dimensiones de caracterización personal, que afectan el rendimiento escolar de estudiantes del centro Educativo Rural Bertrania de básico primario.

Este resultado analizado se prioriza en identificar los factores externo e internos de los estudiantes que afectan el rendimiento escolar. En la caracterización de los docentes se estableció que el $60 \%$ de los docentes son hombre y $40 \%$ son mujeres, según la opinión de los estudiantes, las profesoras son más flexibles y cariñosas que los profesores, también se estableció que el 80\% de los docentes de matemáticas han realizado un pregrado, pero ninguno en el área de matemáticas. Esta es una de las razones por la cual, el docente no se siente cómodos y preparados para desempeñar una buena labor, por lo tanto, sus clases son monótonas y aburridas porque carecen de la didáctica de la enseñanza en ésta área.

Por otro lado, en la caracterización de los estudiantes se pudo comprobar que el $77 \%$ son de género masculino, un 27.7 son de nacionalidad venezolana que presentan una serie de expresiones y manifestaciones culturales diferentes a los niños colombianos. La mayoría de las familias son numerosas con un promedio de 5 hijos.

Un $28 \%$ de los docentes no realiza actividades en clase que los motive, además, un 35\% expresan que las clases son monótonas y aburridoras, donde el docente mantiene el mismo esquema de clase, otro delos factores corresponde a que el 37.7 de ellos no tienen dominio del grupo, presentándose desorden $\mathrm{y}$ en algunos casos agresiones físicas y verbales, por lo cual el $28.8 \%$ de los estudiantes creen que el ambiente escolar no es bueno y agradable, además $44.4 \%$ ve que las condiciones físicas del aula no es las más apropiada para el aprendizaje, también $51.1 \%$ manifiesta que el grupo no se encuentra motivado y además el $46.6 \%$ argumenta no sentirse feliz en las clases de matemáticas.

También se puedo confirmar que $77.7 \%$ de los padres de familia a lo han cursado un grado de básica primaria, lo cual se les dificulta brindarle asesoría y acompañamiento a sus hijos en las tareas, en un $100 \%$ los padres son de estrato $0 \mathrm{y} 1$, esto nos indica que las familias son de bajos recursos económicos, y en alguno de los casos no pueden brindarle las mejores condiciones de vida sus hijo, por otro lado, tampoco tiene las condiciones para comprarle los útiles necesarios para su labor como estudiante. Otro dato importante es que el $50 \%$ de los padres han tenido que sufrir la secuela de la violencia, lo cual afecta la estabilidad de las familias y el bienestar social, psicológico y económico, además en su gran mayoría no tiene un trabajo estable, lo que hace que $50 \%$ de ello coloque a sus hijas a realizar labores en el campo.

\section{Agradecimiento}

Un agradecimiento especial para la Universidad Francisco de Paula Santander, y a toda la planta de docentes que hicieron parte de mi formación a académica, por permitir el convenio con los docentes del Catatumbo para la realización de este proyecto de vida, y hacer realidad mi más grande sueño de ser Magísteres en educación matemática y en especial a Dra. Mawency Vergel Ortega directora del plan de estudios, a la Dra. Laura Yolima Moreno Rozo directora del trabajo de investigación. Matemática. Principalmente a la Doctora Mawency Vergel Ortega, Directora del programa Maestría en Educación Matemáticas. Laura Yolima Moreno Rozo. Directora del Proyecto de investigación. Por su apoyo, su consejo, su entrega a su labor docente, su bondad y humildad que las caracterizan y las hace mejores seres humos.

\section{Referencias}

Anónimo. (sf). Factores determinantes del bajo rendimiento del aprendizaje. Recuperado de: 
Ausubel, (1983), Pág. 55. Teoría del aprendizaje significativo.

Barba Téllez María N (2007) Piaget y L. S. Vigotsky en el análisis de la relación entre Educación y desarrollo

Bandura, A. (1997). Self: Efficacy: The exercise of control. New York: Freeman. Barbero, I., Holgate, F. P., Vila, E. \& Chacón, S. (2007). Actitudes, hábitos de estudio y rendimiento en matemáticas: Diferencias por género. Psicothema, 19(3), 413-442.

Casanova, P. Cruz García-Linares, Manuel J de la Torre \& M de la Villa Carpio, 2005; Eamon, 2005; Jones \& White, (2000). Influencia de las familias en las variables sociodemográficas en estudiantes con bajo rendimiento académico.

Caso-Niebla, Joaquín. (Hernández-Guzmán, Laura Variables que inciden en el rendimiento académico de adolescentes mexicanos, 2007 pág. 489). (2010). Prueba de Autoestima para Adolescentes (2010)

Córdoba S. Osvaldo. (2016). Diseño de un modelo de situación problema, con la unidad facilitadora solidaria llamada belisol1, para fortalecer el pensamiento numérico y variacional,

Castro Pérez M. (2015 pag.03. los ambientes de aula que promueven el aprendizaje, desde la perspectiva de los niños y niñas escolares. Universidad Nacional.

Cornejo Chávez R (2007) Variables y factores asociados al aprendizaje escolar. Una discusión desde la investigación actual. Universidad de Chile

Colombia aprende. La red del conocimiento (2018)

Delval 2000, Aprender en la vida y en la escuela. Pag 70

De la Torre Cruz M. (2005). Influence of family and socio-demographic variables on students with low academic achievement.

Díaz Barriga (2002), Estrategias docente para el aprendizaje significativo

Delia Jara, Hernán Velarde. Factores influyentes en el rendimiento académico de estudiantes del primer año de medicina. (2008)
F. P., Vila, E. \& Chacón, S. (2007). Actitudes, hábitos de estudio y rendimiento en matemáticas: Diferencias por género.

Feldman. Daniel (2004). Imágenes en la historia de la enseñanza: la lámina escolar.

(Shucksmith, 1987) Las estrategias de aprendizaje. Revisión teórica y conceptual

García A. \& Martínez M. (1986) El fracaso Escolar en la región de Murcia

Giroux, Henry A. (1985). Como mejorar la falta de atención en los niños

García A. \& Martínez M. (1986) El fracaso Escolar en la región de Murcia

Gasco Txabarri J. (2016). El empleo de estrategias en el aprendizaje de las Matemáticas en Enseñanza Secundaria Obligatoria

Giroux, Henry A. (1985). Como mejorar la falta de atención en los niños

Georg Gadamer (1993) Verdad y Método.

Hernández-Guzmán, Laura Variables que inciden en el rendimiento académico de adolescentes mexicanos, (2007)

Isaza, L. y Henao, G.C (2012). Actitudes-estilo de enseñanza su relación con el rendimiento académico. International Journal of Psychological Research, ISSN-e 2011- 2079, Vol. $5, \mathrm{~N}^{\mathrm{o}} .1,133-141$.

Isaza, Galeano y Joven (2014) Estilos de enseñanza de los docentes: una apuesta por el desempeño académico de los estudiantes en la Educación Superior. REMO: Volumen XI, Número 26.

Lammers, Onweugbuzie \& Slate, (2001) Proctor, Hurst, Prevatt, Petscher \& Adams, 2006 El existo académicos en función del género, clase, edad hábito de estudio y empleo de estudiantes universitarios.

López Angulo. E (2011. Pág. 113) “política fiscal y estrategia como factor de desarrollo de la mediana empresa comercial sinaloense. Un estudio de caso"

Londoño E. Mariana (2013) Propuesta Pedagógica y didáctica para el área de matemáticas

Mousalli-Kayat, G. (2015). Métodos y Diseños de 
Investigación Cuantitativa. Mérida.

Monereo y Badia. (2013) La enseñanza y el aprendizaje de estrategias en el entorno virtual

Ministerio De Educación Nacional Dirección De Calidad De La Epbm. Ruta De Reflexión y

Mejoramiento Pedagógico "Siempre Día E" octubre De 2015

Moncayo Cabrera, M.A (2018). Determinantes que influyen en el rendimiento académico: un estudio aplicado para Colombia a partir de las pruebas icfes - saber 11

$M^{\mathrm{a}}$ Isabel Barbero García, Francisco Pablo Holgado Tello, Enrique Vila Abad y Salvador Chacón Moscoso. Actitudes, hábitos de estudio y rendimiento en Matemáticas: diferencias por género $(2007$

Navarro, R. (2006) Factores asociados al rendimiento académico. Universidad Cristóbal Colón, México. Revista Iberoamericana de Educación (ISSN: 1681-5653)

Ortiz Granja D. (2015) El constructivismo como teoría y método de enseñanza

Pérez Marqués (UAB, 1999) concepciones sobre el aprendizaje.

Pantoja Ospina. (2013) Modelos de estilos de aprendizaje: una Actualización para su revisión y análisis Pozo, (1989). Teoría del aprendizaje

Ramírez García A, \& Lorenzo Guijarro E. (2012). Desarrollo de la competencia matemática en educación primaria a través de la resolución de tareas

Ruiz. R. Celia (2015). Como mejorar la falta de atención en los niños

Santillana. Reforma integral de educación media y superior

Schmeck (1988); Estilos y estrategias de aprendizaje

Schmeck (1988);(1991). Estrategias de aprendizaje, revisión teórica y conceptual.

Tomado desde http://www.redalyc.org/ pdf $/ 805 / 80531302$.pdf

Strobel L (2015). Ambientes Escolares Saludables

Trevino E y Valdez H (2010) Factores asociados al logro cognitivo de los estudiantes de América
Latina y el Caribe

UNESCO, O. D. (2008). "La educación inclusiva: el camino hacia el futuro". Chile: Unesco.

Velasquez, 2014 Etapas del desarrollo cognitivo de Piaget

Vasco. (2014). Propuesta didáctica de matemáticas. Institution: school of mathematics, yachay tech, yachay city of knowledge, 100119 - Urcuqui Ecuador

h t t p s : / / w w w . r e s e a r c h g a t e.net / publication/327219515_Etapas_del_desarrollo_ cognitivo_de_Piaget

http://www.politecnicometro.edu.co/biblioteca/ primerainfancia/Aprender $\% 20$ en $\% 201 \mathrm{a} \% 20$ vida $\% 20 y \% 20$ en $\% 201 \mathrm{a} \% 20$ escuela.pdf

h t t p s : / / e n . c a 1 a m e o . c o m / $\mathrm{read} / 005023474 \mathrm{~d} 70 \mathrm{c} 4 \mathrm{afbd} 830$

h t t p s : / / e n . c a 1 a m e o.c o m / $\mathrm{read} / 0047058168 \mathrm{e} 6 \mathrm{aa} 68 \mathrm{ce} 8 \mathrm{~d} 0$

http://aprende.colombiaaprende.edu.co/es/ search/site/indice $\% 20$ sintetico $\% 20$ centro $\% 20$ educativo $\% 20$ rural $\% 20$ Bertrania

https://www.universidadviu.com/factoresdeterminantes-del-bajo-rendimiento-en-elaprendizaje/, 2018

http://ridum.umanizales.edu.co:8080/jspui/ bitstream/6789/2613/1/Monsalve_Henry_De_ Jes\%C3\%BAs_2016.pdf

http://ficus.pntic.mec.es/fheb0005/Hojas_varias/ Material_de_apoyo/Estrategias $\% 20 \mathrm{de} \% 20$ Polya pdf

http://www.ub.edu/dppsed/fvillar/principal/pdf/ proyecto/cap_05_piaget.pdf

https://www.academia.edu/12834496/ TEOR\%C3\%8DA_Y_PRINCIPALES PERSONAJES_CONSTRUCTIVISTAS

http://repository.lasalle.edu.co/bitstream/ handle/10185/20691/10091033_2016.pdf

https://rieoei.org/historico/investigacion/512Edel. PDF

http://eds.a.ebscohost.com/eds/pdfviewer/ pdfviewer?vid=1\&sid=8b8d41 de-642d-4eb28671-d540a6761940\%40sdc-v-sessmgr01 